\title{
Conjuntivite neonatal com ênfase na sua prevenção
}

\section{Neonatal conjunctivitis with emphasis on its prevention}

Angelo Ferreira Passos ${ }^{1}$, Fernanda Spinassé Agostini ${ }^{2}$

\begin{tabular}{|l}
\hline$\underline{\text { RESUMO }}$ \\
Nos últimos tempos, a necessidade da utilização de medicamentos para prevenção da \\
conjuntivite neonatal (CN) passou a ser questionada em alguns países desenvolvidos, \\
devido ao elevado nível de assistência pré-natal. Ao contrário, no Brasil, embora não \\
haja dados oficiais sobre sua ocorrência, vários trabalhos recentes comprovam elevada \\
prevalência da infecção genital em mulheres em idade fértil e em gestantes. Isso, aliado \\
ao fato de que o índice de transmissão da infecção genital por clamídia e gonococo, da \\
mãe infectada para o recém-nascido é de 30 a 50\%, leva à conclusão de que a profilaxia \\
medicamentosa está mais que justificada. A CN implica em importante potencial de \\
complicações locais e sistêmicas, além da necessidade de exames laboratoriais para \\
seu diagnóstico etiológico. Por isso, constitui importante problema de saúde pública, \\
negligenciado no Brasil, onde não há padronização do método de prevenção. Embora o \\
uso do nitrato de prata pareça ainda ser o método oficial, seu uso tem sido questionado \\
devido à incompleta proteção contra clamídia, principal agente da conjuntivite neonatal \\
nos dias atuais, e pela frequente ocorrência de conjuntivite química. Por isso, tem sido \\
substituído por outros agentes, como a eritromicina, a tetraciclina, além de outros anti- \\
bióticos. A superioridade da Iodopovidona em relação a esses antibióticos, nos vários \\
quesitos analisados, tem sugerido que esse é o mais adequado entre os produtos, testa- \\
dos até o momento, para prevenção da CN.
\end{tabular}

Descritores: Oftalmia neonatal; Conjuntivite; Prevenção primária; Nitrato de prata; Doenças sexualmente transmissíveis; Chlamydia trachomatis; Neisseria gonorrhoe; Povidona-Iodo

\footnotetext{
'Professor Associado 3; Chefe do Serviço de Oftalmologia do Hospital Universitário Cassiano Antônio de Moraes (HUCAM), Universidade Federal do Espírito Santo - UFES - Vitoria (ES), Brasil;

${ }^{2}$ Acadêmica do $12^{\circ}$ período do Curso de Medicina; Estagiária do Serviço de Oftalmologia Hospital Universitário Cassiano Antônio de Moraes (HUCAM), Universidade Federal do Espírito Santo - UFES - Vitoria (ES), Brasil.
} 


\section{INTRODUÇÃO}

$\mathbf{S}$ egundo o Ministério da Saúde (MS), Conjuntivite Neonatal $(\mathrm{CN})$ é definida como uma conjuntivite purulenta do recém-nascido, no primeiro mês de vida, usualmente contraída durante o seu nascimento, a partir do contato com secreções genitais maternas contaminadas $^{(1)}$.

A CN foi um grande problema de saúde pública durante séculos, em todo o mundo. No final do século XIX, na Europa, sua prevalência entre os nascidos vivos, em maternidades e hospitais, excedia a taxa de $10 \%$, produzindo lesão corneana em $20 \%$ e cegueira em $3 \%$ dos acometidos. Era a principal causa de cegueira infantil, sendo responsável por 60 a $73 \%$ dos casos nas instituições para crianças cegas $^{(2)}$. A epidemiologia desta condição mudou radicalmente com a introdução do método de Credé, em 1881, que consistia na aplicação de uma gota de solução aquosa de nitrato de prata (NP) a $2 \%$, após a limpeza dos olhos. Esse procedimento reduziu dramaticamente a incidência da CN, não só na Europa, mas em todo o mundo ${ }^{(3)}$.Posteriormente, a solução de NP a 1\% passou a ser o método de Credé, por ser menos irritante que a $2 \%{ }^{(4)}$.

\section{Etiologia e tratamento}

Além dos agentes microbianos, o produto utilizado na prevenção pode também causar a CN. Essa conjuntivite química é relatada em 10 a $100 \%$ dos neonatos que são submetidos aos agentes profiláticos. Pode, até mesmo, ocorrer com a utilização de eritromicina ou tetraciclina, mas é comumente associada à profilaxia com o $\mathrm{NP}^{(5)}$. Costuma apresentar-se como uma conjuntivite purulenta, discreta, que, em geral, se resolve espontaneamente em até 48 horas $^{(6)}$.

A grande maioria dos casos de $\mathrm{CN}$ infecciosa é adquirida durante a passagem pelo canal do parto e reflete as doenças sexualmente transmissíveis (DST) presentes na comunidade $^{(7)}$. Com relação aos agentes microbianos, no Manual DST AIDS do MS de 2006 são reportados como os mais importantes a Neisseria gonorrhoeae (NG) e a Chlamydia trachomatis (CT), sendo a CT considerada a principal bactéria implicada na gênese da $\mathrm{CN}$, em especial nos países industrializados ${ }^{(1)}$. Enquanto nos países em desenvolvimento a incidência de NG e CT estava entre cinco e 50 por 1000 nascidos vivos, para ambos os agentes, nos desenvolvidos, a incidência da NG era de 0,1 a 0,6 e a de CT era de 5 a 60 por 1000 nascidos vivos ${ }^{(1)}$.

Outras bactérias também podem causar a $\mathrm{CN}$. Depois da CT, por ordem de frequência são relatadas as seguintes bactérias como agentes da CN: Streptococcus viridans, Staphylococcus aureus, Haemophilus influenzae, Streptococcus do grupo D, Moraxella catarhalis, Escherichia coli e outros bastonetes gram-negativos, e a $\mathrm{NG}^{(8)}$. Também é relatado que, de todas essas bactérias, a isolada mais frequentemente é o S. aureus, mas como o índice de colonização da conjuntiva dos neonatos sem conjuntivite, por esse agente, também é alto, variando entre 1 a $28 \%$, a patogênese desse estafilococo na CN é incerta ${ }^{(9,10)}$.

A CN viral é quase exclusivamente herpética ${ }^{(7)}$, tendo em vista o grande número de agentes que se podem apresentar de forma semelhante, a etiologia da $\mathrm{CN}$ não pode ser definida com base apenas na clínica, fazendo-se necessário o uso de métodos laboratoriais ${ }^{(1)}$. Talvez a justificativa mais importante para a necessidade de identificação do agente etiológico é que, ao contrário da conjuntivite do adulto, a $\mathrm{CN}$ implica em maior risco de complicações locais e significativo potencial de complicações sistêmicas, de gravidade dependente do microorganismo, necessitando de pronto tratamento direcionado ao agente específico.

Assim sendo, deve ser feito inicialmente o exame direto do raspado conjuntival, com as colorações de Giemsa e Gram, respectivamente, para CT e para outras bactérias (inclusive a NG). Se o Giemsa for negativo, mas persistir a suspeita de clamídia, podem ser feitos os testes de imunofluorescência ou PCR ${ }^{(11,12)}$. Se a suspeita for de herpes, o exame indicado pode ser o Papanicolaou ${ }^{(10)}$. A cultura e o TSA devem complementar o exame direto ${ }^{(10)}$.

O início da terapia antibacteriana deve ser orientado pelo imediato exame direto, sendo sua manutenção ou alteração definida pela cultura e $\operatorname{TSA}^{(7)}$. De acordo com o Manual DST/AIDS - 2006, do MS, na indisponibilidade desses exames, "toda oftalmia neonatal deve receber tratamento para NG e CT" (1).

Enquanto para O'Hara (1993) ${ }^{(7)}$ outras bactérias podem ser tratadas topicamente, $\mathrm{o}$ tratamento deve ser sistêmico para a $\mathrm{NG}^{(1,13)}$ e $\mathrm{CT}^{(13,14)}$, sendo bastante utilizados e seguros, respectivamente, o ceftriaxone e a eritromicina. O Herpes simples também deve ser tratado sistemicamente ${ }^{(7,13)}$. No Manual DST /AIDS do MS de $2006^{(1)}$ é sugerido que no caso de resposta terapêutica não satisfatória ao tratamento da NG, se deve considerar a hipótese de infecção simultânea por CT.

\section{DST no Brasil e no mundo}

Segundo dados da Organização Mundial de Saúde (OMS), de 2001, a infecção genital por CT é a DST bacteriana mais comum em todo o mundo ${ }^{(15)}$. No Brasil, 
estudos realizados, de 2000 a 2008, nas cidades de Campinas, Salvador, Fortaleza e Vitória, com mulheres sexualmente ativas, na faixa etária de 12 a 49 anos, atendidas em serviços de atenção básica e planejamento familiar, mostraram prevalência de CT variando entre 7,4 e $17,1 \%$ e de gonococo entre 0,5 e $2 \%{ }^{(16,20)}$. Em gestantes, estudos realizados em Manaus, Fortaleza, Goiânia, Rio de Janeiro, São Paulo, Porto Alegre e Belo Horizonte, nos anos de 1999 a 2005, mostraram prevalência de CT entre 9,4 e $24,4 \%$ e de gonococo de $1,5 \%{ }^{(21,23)}$.

Muitas vezes, a infecção por CT é assintomática, o que justifica a necessidade de um rastreio para a mesma ${ }^{(23,24)}$. Nos EUA, a triagem para infecção nas gestantes já é recomendada há mais de 10 anos ${ }^{(25,26)}$.

A infecção por gonococo, conforme exposto, é menos frequente, porém é ainda um problema em países desenvolvidos, onde afeta principalmente adolescentes, e é um problema grave em países subdesenvolvidos ${ }^{(27,28)}$. De acordo com esse fato, a OMS, em documento publicado em 2001, demonstrou que a $\mathrm{CN}$ ainda era uma das principais causas de cegueira em países pobres na África e de outros continentes ${ }^{(29)}$.

Segundo o MS, o risco de transmissão da infecção genital da mãe para o recém-nascido, causando conjuntivite, está entre 30 e 50\%, para CT e NG, por ocasião do parto vaginal ${ }^{(1)}$. No parto cesáreo, o risco é menor, mas existe, principalmente no caso de prolongado tempo de rotura de membranas ${ }^{(13,30)}$.

\section{Profilaxia da CN no Brasil e no Mundo}

A partir do final do século XIX, tendo em vista a alta prevalência da infecção genital pela NG, o método de Credé teve inquestionável importância na prevenção da $\mathrm{CN}$ e da cegueira, na Europa e em todo o mundo. No Brasil, ele foi regulamentado em 1977, pelo Decreto-Lei $9713^{(31)}$, e posteriormente, complementado pelo Decreto-Lei $19941^{(32)}$,que normatizou a operacionalização do método.

Até o final do século XX, o método de Credé foi o de escolha para a profilaxia de conjuntivite neonatal em todo o Brasil ${ }^{(33)}$, tendo sido ainda recomendado na última edição do Manual DST /AIDS do MS, $2006^{(1)}$.

O NP é usado como antisséptico e adstringente. Seu poder germicida deve-se à combinação do íon prata com certos grupamentos das proteínas dos micro-organismos, levando à sua desnaturação, com a consequente ruptura e morte do germe. O NP destrói a maioria dos micro-organismos na concentração de $0,1 \%$; concentrações menores têm propriedades bacteriostáticas ${ }^{(33-35)}$.

A Farmacopéia Americana estabelece que: a concentração de solução de NP para uso oftálmico não deve ser inferior a $0,95 \%$ e nem superior a $1,05 \%$; o $\mathrm{pH}$ da solução quimicamente pura a $1 \%$ deve estar entre 4,5 e 6; deve ser envasada em frasco de vidro neutro, de cor escura e provido de dispositivo conta-gotas, que deve ser mantido bem fechado, ao abrigo do calor e da luz; o tempo adequado de troca da solução é de 24 a 48 horas. É ainda ressaltado que a solução de NP é instável. Sua concentração aumenta pela evaporação e se modifica pela ação da luz, originando uma solução fortemente cáustica. $\mathrm{O}$ calor dissocia suas moléculas, formando radicais livres. Daí, a necessidade de armazená-la durante o tempo correto e em condições adequadas ${ }^{(36)}$.

Mas o fato é de que o uso do NP há muito tem sido questionado, devido ao seu efeito irritativo, além de sua incompleta proteção contra CT, o principal agente causador da $\mathrm{CN}$ nos dias atuais ${ }^{(37,38)}$.

Pelo fato de ser irritativo e, talvez, também, por não estar disponível para venda, em farmácias, levantamentos realizados no período de 1975 a 1999, por vários autores, em capitais de alguns estados do Brasil ${ }^{(39,43)}$, mostraram que o método de Credé não era utilizado em grande porcentagem das maternidades, sendo substituído pelo vitelinato de prata a $10 \%\left(\right.$ Argirol $\left.^{\circledR}\right)$. Os estudos mostraram também que o NP era frequentemente utilizado de forma supostamente incorreta, em grande parte das casas de parto, inclusive no que diz respeito ao tempo de armazenamento da solução, visto ser necessária sua renovação, pelo menos, a cada 48 horas, de acordo com a Farmacopeia dos Estados Unidos ${ }^{(36)}$. Ainda, em levantamento bem mais recente, do mês de março de 2007, realizado em cinco maternidades da cidade de Florianópolis, foi encontrado que, em três delas, a profilaxia da $\mathrm{CN}$ era realizada com o vitelinato de prata, e nenhuma utilizava o NP ${ }^{(44)}$.

O vitelinato de prata é um antisséptico de ação lenta e persistente, capaz de alterar a lisozima da lágrima e, embora pouco irritante, é muito menos eficaz que o NP, conforme estudo experimental realizado em coelhos ${ }^{(45)}$. Não foi encontrada nenhuma recomendação do uso do vitelinato de prata para a profilaxia da $\mathrm{CN}$, pelo MS ou por qualquer outra instituição de saúde pública ou privada. Assim sendo, não parece haver qualquer fundamentação para seu uso, sendo, portanto, um estranho equívoco, a substituição que vem sendo feita há muitos anos, em tantos serviços do Brasil, do NP pelo vitelinato de prata.

Em um estudo realizado pelo Setor de Farmacovigilância do Centro de Vigilância Sanitária, da Secretaria de Saúde do Estado de São Paulo, entre março de 2003 e março de 2004, foram relatados 622 casos de conjuntivite química, pelos profissionais de saú- 
de de 11 grandes hospitais daquele Estado. Apesar da conjuntivite química, geralmente, ter um efeito colateral não muito sério e autolimitado, ela causa muita ansiedade nas mães. É interessante que apenas 6 dos 11 hospitais relataram a ocorrência da conjuntivite química, após o uso tópico do NP. Os outros cinco hospitais quando interrogados por que não relataram a conjuntivite, como uma reação adversa do uso da medicação, responderam que os profissionais de saúde daquelas instituições consideravam como "normal" a conjuntivite relacionada ao uso do NP ${ }^{(46)}$.

É importante ressaltar que, embora o NP a 1\% seja realmente irritativo para os olhos, é possível que boa parte dos casos de conjuntivite química, mormente os mais intensos, esteja associado à concentração mais elevada da solução, conforme também sugerem Mathieu, $1958^{(47)}$ e Giffin, $1967^{(48)}$, principalmente considerando que grande parte dos serviços não armazena o medicamento da maneira e pelo tempo recomendados ${ }^{(39,432)}$.

Instilações acidentais de solução de NP mais concentrada (10 a $20 \%$ ) podem causar ulceração conjuntival e corneana, com sequela cicatricial grave, e catarata ${ }^{(10)}$, tendo sido relatados casos importantes por alguns autores citados por Giffin, em $1967^{(48)}$.

Pelo exposto, houve quem passasse a substituir o NP por antibióticos, dentre os quais a tetraciclina e a eritromicina parecem ter sido os mais utilizados, com base no fato da CT estar incluída no seu espectro de ação, e também pela sua esperada ação contra bactérias grampositivas, frequentemente relacionadas com a $\mathrm{CN}$, e contra cepas de NG. No entanto, esses agentes não se mostraram mais eficazes contra a CT que o NP, pelo menos quando utilizados da mesma maneira, ou seja, em aplicação única $^{(12,49-52)}$. Além disso, os antibióticos citados levaram à geração de resistência bacteriana e à consequente ocorrência de epidemias, relacionadas com germes resistentes, como estafilococos ${ }^{(53,54)}$. Assim, restou, como única vantagem desses antibióticos, em relação ao NP, a menor ocorrência de conjuntivite química ${ }^{(6)}$.

Apesar do exposto, ainda em publicação relativamente recente, de 2006, o MS orientava a profilaxia da $\mathrm{CN}$ da seguinte forma: "Nitrato de prata a 1\% (Método de Credé), ou eritromicina a $0,5 \%$ (colírio), ou tetraciclina a $1 \%$ (colírio), em aplicação única, na $1^{a}$ hora após o nascimento" (1).

Ao que tudo indica, também parece não haver uma prática comum adotada pela comunidade internacional para a profilaxia da CN. Enquanto no Reino Unido e na Austrália não se realiza mais a profilaxia, na maioria dos estados dos Estados Unidos da América ela é obrigatória por lei ${ }^{(55)}$.

Segundo relato de Dipiro, em $2002^{(56)}$, a Academia Americana de Pediatria recomendava o uso de NP a $1 \%$, tetraciclina a $1 \%$ ou eritromicina a $0,5 \%$, mas salientava que, destes agentes, o NP era o único com eficácia documentada na prevenção da oftalmia causada por NG produtora de penicilinase. Foi relatado também que em aproximadamente $2 \%$ das crianças sob risco de infecção ocorre falha na profilaxia com os antibióticos recomendados ${ }^{(56)}$. Por isso, as crianças que nasciam de mães comprovadamente infectadas deveriam receber também ceftriaxona sistêmica ${ }^{(56,57)}$.

O Guia de DST publicado pelo Centers for Disease Control and Prevention (CDC) dos Estados Unidos, de $2002^{(58)}$ recomendava os três produtos, mas o de $2006{ }^{(26)}$ mencionava apenas os dois antibióticos.

Em agosto de 2009, devido a uma mudança nos fabricantes da pomada de eritromicina, houve escassez do produto nos EUA. Além disso, no mesmo mês, o CDC enviou comunicação aos médicos americanos, informando que a pomada de tetraciclina a $1 \%$ e a solução de NP a $1 \%$ não estavam mais disponíveis para uso nos EUA ${ }^{(59)}$. Assim sendo, muitos hospitais passaram a utilizar a pomada de gentamicina para a profilaxia da CN. No entanto, esse medicamento causou reações oculares nos neonatos, como edema, dermatite e bolhas na pele. Por isso, e pela falta da eritromicina, o CDC passou a recomendar o colírio de azitromicina, para a profilaxia da $\mathrm{CN}$, apesar de não haver estudos que comprovassem a eficácia do produto para tal, mas apenas pela sua analogia com a eritromicina, não disponível ${ }^{(60)}$.

Em março de 2010, o FDA informou que o problema da escassez da pomada de eritromicina já havia sido resolvido. Com isso, o CDC reafirmou, na mesma data, que a pomada de eritromicina era o único agente recomendado para profilaxia da $\mathrm{CN}$ disponível nos EUA ${ }^{(60)}$.

Em publicação de 2009, a Sociedade Canadense de Pediatria ${ }^{(61)}$, apesar do decréscimo da prevalência da NG no Canadá e de recomendar triagem para infecções por NG e CT, durante a gestação, pré e pós-natal, faz recomendação de que todos os neonatos recebam profilaxia para a $\mathrm{CN}$ com NP, tetraciclina ou eritromicina. Informa essa Sociedade que a infecção pela NG corresponde a menos de $1 \%$ dos casos registrados de $\mathrm{CN}$ no Canadá e nos EUA, enquanto pela $\mathrm{CT}$ varia de $2 \mathrm{a}$ $40 \%$ e que os casos de $\mathrm{CN}$ por esses patógenos diminuíram nas últimas duas décadas, como resultado da redução da prevalência dessas infecções genitais na população geral, bem como da instituição da triagem na rotina pré-natal ${ }^{(61)}$. 


\section{Sobre a Iodopovidona}

A partir de 1990, a iodopovidona (PVPI) passou a ser vista como um agente potencial na profilaxia da $\mathrm{CN}$.

Benevento et al. ${ }^{(37)}$, partindo do fato de que essa substância era efetiva na prevenção das infecções relacionadas com cirurgias intraoculares, por atuar de maneira significativa na flora conjuntival de adultos ${ }^{(62-64)}$, resolveram avaliar sua ação sobre a flora relacionada com a $\mathrm{CN}$, assim como sua toxicidade para a superfície ocular. Procuraram então determinar a sensibilidade da $\mathrm{NG}$ (quatro cepas), CT (duas cepas) e H. simplex tipo II (uma cepa), utilizando diferentes concentrações da solução de iodopovidona $(5 \%, 1 \%$ e $0,1 \%)$, com o tempo de exposição de 1 minuto. Ficou demonstrado que a iodopovidona é efetiva contra gonococo e herpes em concentrações tão baixas quanto $0,1 \%$ e é contra clamídia nas concentrações de 1 e de $5 \%{ }^{(37)}$.

Em 1994, foi realizado um estudo piloto, para avaliação da iodopovidona na prevenção da CN, em comparação com outros agentes. Foi realizada cultura das conjuntivas dos dois olhos de 100 neonatos, após $30 \mathrm{mi}-$ nutos de vida. Num olho foi aplicado iodopovidona a $2,5 \%$, no outro NP a $1 \%$ ou eritromicina a $0,5 \%$. Nova cultura foi realizada com 4 horas de vida, ao mesmo tempo em que os olhos também foram avaliados quanto a reações tóxicas. Para medir a efetividade dos medicamentos, foram comparados o número de bactérias formadoras de colônias e o número de espécies de cada cultura. Foi observado que os três agentes reduziram o número de colônias e de espécies da conjuntiva dos recém-nascidos, sendo que o primeiro agente atingiu o melhor nível de significância estatística $(\mathrm{p}<0,05$ para número de colônias e $\mathrm{p}<0,002$ para número de espécies) e o último, o pior. O estudo também permitiu concluir que a iodopovidona a $2,5 \%$ é relativamente não tóxica. Após 24 horas da aplicação, o NP produziu uma toxicidade com score de 2 ou mais (geralmente, indicando hiperemia conjuntival moderada a severa, edema palpebral e descarga purulenta) em $10 \%$ dos casos. Os outros dois agentes produziram resposta similar em apenas 1 a $2 \%$ dos casos ${ }^{(38)}$. No pré-operatório de cirurgias oftalmológicas, a solução de iodopovidona é geralmente utilizada na concentração a $5 \%$. No entanto, Isenberg et al. (1994) demonstraram que tal concentração ocasionalmente produziu hiperemia conjuntival em alguns neonatos, enquanto a solução a $2,5 \%$ não se mostrou irritativa ${ }^{(38)}$.

Foi realizado também um ensaio controlado, envolvendo 3117 neonatos, nascidos em um período de 30 meses, em um hospital do Kênia. Cada recém-nascido recebeu profilaxia para a $\mathrm{CN}$, em ambos os olhos, de forma aleatória, sendo utilizado iodopovidona a 2,5\%, $\mathrm{NP}$ a $1 \%$ ou eritromicina a $0,5 \%$. A incidência geral de conjuntivite foi menor no grupo que recebeu profilaxia com a solução de iodopovidona $(13,1 \%)$, quando comparada com os demais grupos (17,5\% para o NP, $p<0,001$; $15,2 \%$ para a eritromicina, $\mathrm{p}=0,01)$. Além disso, a iodopovidona foi mais efetiva contra clamídia do que o NP $(p<0,001)$ e do que a eritromicina $(p=0,008)$. Neonatos que receberam profilaxia com NP e eritromicina tiveram, respectivamente, $92 \%$ e $34,5 \%$ mais conjuntivite por clamídia do que os que foram tratados com iodopovidona. Ocorreu $9,7 \%$ de conjuntivite não infecciosa no grupo que recebeu profilaxia com iodopovidona, comparado com $13,9 \%$ no grupo do NP $(\mathrm{p}<0,001)$ e $13,3 \%$ no grupo da eritromicina $(\mathrm{p}=0,004)^{(65)}$.

Em outubro de 2000, em um ensaio clínico, Najafi et al. ${ }^{(66)}$ compararam um grupo de 475 neonatos em que instilaram uma gota de iodopovidona a 2,5\% em ambos os olhos de um grupo controle de 385 neonatos que não recebeu profilaxia alguma. No primeiro grupo, secreção ocular foi observada em 14 neonatos $(2,94 \%)$, dentre os quais seis $(1,26 \%)$ apresentaram culturas negativas e oito $(1,68 \%)$ culturas positivas, sendo quatro $(0,84 \%)$ para S. epidermidis, três $(0,63 \%)$ para S. aureus e um $(0,21 \%)$ para Pseudomonas. No grupo controle, secreção foi observada em $42(10,9 \%)$ neonatos, sendo que nove $(2,33 \%)$ apresentaram culturas negativas e 33 $(8,57 \%)$ culturas positivas, sendo $15(3,89 \%)$ para $S$. epidermidis, 14 para $S$. aureus ( 3,63\%), dois para Enterobacter $(0,52 \%)$ e dois para E. coli $(0,52 \%)$. Ressaltando a eficácia da Iodopovidona, os autores consideram que os organismos encontrados nos olhos dos neonatos após a administração da solução foram prováveis contaminantes do meio ambiente, que se instalaram nos olhos após ter cessado o efeito da iodopovidona instilada ${ }^{(66)}$.

Ensaio clínico randomizado realizado durante os anos de 2004 e 2005 comparou o resultado da iodopovidona a $2,5 \%$, comparado com a eritromicina a $0,5 \%$ e nenhum medicamento, na prevenção da $\mathrm{CN}$, em trezentos e trinta neonatos em três grupos de 110. A ocorrência de conjuntivite foi constatada em 52 neonatos, sendo que $9 \%$ dos infectados haviam recebido iodopovidona, $18,4 \%$ eritromicina e $22,5 \%$ nenhum tratamento. Concluiu-se pela eficácia da iodopovidona, sendo essa significativamente maior que a da eritromicina ${ }^{(67)}$.

Foi demonstrado também que a iodopovidona é ativa não apenas contra os agentes bacterianos mais importantes da CN como a CT, NG e outras bactérias, 
mas também contra fungos ${ }^{(37)}$ e contra o vírus da imunodeficiência humana (Harbiston 1989, apud Benevento 1990) ${ }^{\left({ }^{(37}\right)}$, além dos herpes vírus I e II ${ }^{(37)}$. Segundo Houang e Prince não foi demonstrada resistência bacteriana à iodopovidona ${ }^{(68,69)}$.

Outros estudos foram realizados visando obter maiores informações sobre a segurança do uso da iodopovidona. Assim, Najafi et al. ${ }^{(66)}$ estudaram parâmetros como $\mathrm{pH}$, capacidade de autopreservação, esterilidade e estabilidade clínica da solução de iodopovidona a 2,5\%. Concluíram que o pH da solução deve ser mantido em torno de 5 , semelhante ao $\mathrm{pH}$ da lágrima, usando-se hidróxido de sódio a $0,1 \mathrm{~N}$ e ácido cítrico a $0,5 \%$, pois, dessa forma, a iodopovidona é mais estável. Também concluíram que os recipientes de vidro conservam o produto por mais tempo (35 meses) se comparados aos de plástico ( 9 meses). Foi constatado ainda que a solução de iodopovidona a 2,5\% não necessita de conservantes para ser preservada ${ }^{(66)}$

Richter et al., preocupados com a influência que o colírio de iodopovidona pudesse exercer sobre a função tireoideana, realizaram um ensaio clínico randomizado com 69 neonatos, instilando uma gota de iodopovidona a $1,25 \%$ em cada olho. Após a aplicação da iodopovidona, tanto a excreção urinária de iodo quanto os níveis de TSH sérico permaneceram em níveis fisiológicos, demonstrando que a iodopovidona não exerceu efeito sobre a função tireoidiana. O estudo foi realizado com solução de iodopovidona a 1,25\%, pois, segundo os autores, nessa concentração, a iodopovidona é mais bem tolerada, além do que parece ser uma concentração adequada para a prevenção de conjuntivite Neonatal ${ }^{(70)}$.

Apesar do exposto, parece haver certa controvérsia sobre a conveniência do uso da iodopovidona na profilaxia da CN. Em publicação de 2009, a Sociedade Canadense de Pediatria não recomendava o uso da iodopovidona para a profilaxia da $\mathrm{CN}$, considerando que estudos confirmatórios deveriam ser realizados antes que esse produto pudesse ter seu uso recomendado na rotina (61). Em publicação de 2009, o CDC não recomendava o uso da iodopovidona pelo risco de utilização da sua forma detergente, de uso mais comum, o que poderia ser perigoso ${ }^{(60)}$. Keenan JD, em $2010{ }^{(71)}$, apenas coloca a iodopovidona como uma alternativa de uso na falta da eritromicina, aparentemente pelo fato de ser ela mais barata que a azitromicina.

Não existem dados oficiais sobre a utilização da iodopovidona no Brasil. Em publicação de 2000, a Agência Nacional de Vigilância Sanitária (ANVISA) ${ }^{(72)}$, órgão vinculado ao MS, fala da conveniência do uso da iodopovidona para profilaxia da CN (“o NP a 1\% não atua sobre a clamídia e tem sido substituído pela iodopovidona em solução ocular a 2,5\%"). Nova citação é feita em 2005, pela mesma Agência, na primeira edição do Formulário Nacional (aprovado pela Resolução RDC no 222 , de 29 de julho de 2005) ${ }^{(73)}$. No entanto, o manual de DST/AIDS de 2006, também do MS, não menciona a possibilidade do seu uso ${ }^{(1)}$. Enquanto isso, tem-se a informação extraoficial da sua utilização em algumas maternidades do país, entre as quais, duas de Florianópolis ${ }^{(44)}$.

Em fevereiro de 2005, no Serviço de Oftalmologia do Hospital Universitário Cassiano Antônio de Moraes (Hucam),em Vitória (ES), foi atendido um caso de queimadura ocular em consequência da utilização de método de prevenção da $\mathrm{CN}$, em maternidade do estado do Espírito Santo, onde era utilizado o Credé. Esse fato desencadeou a elaboração de uma monografia de final de Residência em Oftalmologia, seguida de uma publicação ${ }^{(74)}$ e de um projeto de extensão universitária, visando à mudança do protocolo de prevenção da CN. A mudança deveria começar pela maternidade do Hospital Universitário, seguida das demais maternidades públicas e privadas do Estado, com a pretensão de que se estendesse aos demais estados da União. A mudança implicaria basicamente na adoção oficial da iodopovidona a $2,5 \%$, em substituição a qualquer outro produto.

Esse projeto se iniciou em agosto de 2007, com um levantamento dos métodos de prevenção da $\mathrm{CN}$ nas maternidades na cidade de Vitória(ES). O procedimento inicial teve como objetivo a confirmação de que o mesmo estava justificado. Na ocasião foi encontrado nas seis instituições visitadas (três vinculadas ao SUS e três privadas) quatro que utilizavam o vitelinato de prata a $10 \%$ (Argirol ${ }^{\circledR}$ ) e duas o NP a 1\%. Esse resultado mostrou um quadro não muito diferente dos levantamentos realizados em outras diversas capitais brasileiras, vários anos atrás ${ }^{(34,39-43)}$ e, juntamente com os dados obtidos em levantamento também realizado em 2007, em Florianópolis ${ }^{(44)}$, revelam que é bem provável que nos demais estados brasileiros ainda não haja nenhuma padronização quanto ao método de prevenção da $\mathrm{CN}$.

Depois de muito trabalho, incursões em várias frentes, mas tendo como foco principal os neonatologistas responsáveis pelos berçários e a Secretaria Estadual de Saúde (SESA), conseguiu-se, a princípio, a implantação do uso da iodopovidona em algumas maternidades públicas e privadas de cidade. Por fim, após extenso estudo, visando à comprovação das argumentações apresenta- 
das no projeto de extensão, no final de 2009, o Setor de Farmácia da SESA emitiu um parecer favorável à mudança do protocolo. Esse parecer resultou na publicação, em 27 de janeiro de 2010, de uma portaria, pelo Secretário Estadual de Saúde, determinando o uso da iodopovidona a $2,5 \%$, para profilaxia da $\mathrm{CN}$, em todas as maternidades da Rede Pública e Conveniada do Estado, em detrimento do Nitrato de Prata a 1\%, até então utilizado ${ }^{(75)}$.

\section{Comentários finais}

A CN é uma importante doença ocular em neonatos, sendo considerada uma condição potencialmente séria, tanto pelos efeitos locais, quanto pelo risco de disseminação sistêmica. Por isso e pelo fato de que o seu diagnóstico etiológico não é simples, havendo necessidade da utilização de exames complementares para que seu tratamento seja realizado de maneira segura, o que resulta em custos significativos, constitui importante problema de saúde pública, negligenciado em nosso meio.

Não se encontraram dados oficiais sobre a prevalência da $\mathrm{CN}$ no Brasil, mas apenas dois relatos extraoficiais, de Recife, utilizando a mesma casuística, que revelam acometimento de 0,7 e de $3,0 \%$ dos recém-nascidos ${ }^{(76,77)}$. Dessa forma, não se tem uma noção razoável sobre sua verdadeira ocorrência em nosso meio. No entanto, relatos recentes sobre a elevada prevalência de DST em mulheres em idade fértil e em gestantes ${ }^{(16-23)}$, além das precárias condições de controle pré-natal e do elevado índice de transmissão da infecção da mãe para o $\mathrm{RN}^{(1)}$ permitem inferir que a prevalência da $\mathrm{CN}$ seja elevada, o que justifica a utilização de método preventivo.

Os estudos existentes até o momento parecem deixar bem estabelecidas algumas das propriedades da Iodopovidona: espectro de ação muito amplo, sendo um produto ativo contra todos os possíveis agentes da $\mathrm{CN}$, nesse aspecto, com nítida vantagem sobre os demais produtos utilizados até o momento, inclusive em sua atividade contra a CT, o agente presumidamente mais frequente na $\mathrm{CN}$ não induz resistência microbiana; baixa toxidade local na concentração proposta; provável ausência de toxicidade sistêmica; estabilidade; autopreservação; autoesterilidade; disponibilidade; baixo custo; torna a superfície ocular amarronzada, por alguns minutos, o que serve como indicador de uma aplicação correta ${ }^{(37,38,65-70,78)}$. Tais propriedades permitem afirmar, como havia sido feito no Congresso da Sociedade Austríaca de Oftalmologia, já em $2000{ }^{(79)}$, e, por Schaller, em 2001, no Bullettin of World Health Organization ${ }^{\left({ }^{(0)} \text {, }\right.}$ que a iodopovidona é o produto de escolha na prevenção da CN, por ser ela, diante das evidências, o melhor dentre os produtos testados até o momento.

Apesar de todos os dados favoráveis à conveniência do uso da iodopovidona, entidades respeitáveis, como o CDC, o FDA e a Sociedade Canadense de Pediatria não a têm recomendado ${ }^{(26,60,61)}$. No entanto, são indicados ainda o $\mathrm{NP}^{(61)}$ e antibióticos ${ }^{(26,60)}$ que, em relação à iodopovidona, repetindo, nenhum trabalho foi encontrado que tenha mostrado sua superioridade, em qualquer dos quesitos avaliados ${ }^{(37,38,53,54,65-70,78)}$, exceto quanto ao custo, em que a eritromicina foi mais vantajosa, em levantamento feito nos EUA $^{(71)}$. Até mesmo a azitromicina, que, ao que tudo indica, ainda não foi testada, chegou a ser indicada ${ }^{(60)}$.

Foram encontrados três argumentos contrários a utilização da iodopovidona. O primeiro, da Sociedade Canadense de Pediatria, de que não há estudos suficientes para que seu uso seja indicado ${ }^{(61)}$. O segundo, encontrado em uma publicação feita através do CDC dos EUA, de que se pode confundir e usar a solução do produto com detergente ${ }^{(60)}$, e o terceiro de que a iodopovidona não é ativa contra vírus, porque o vírus penetra na célula, que aparentemente o protege contra a iodopovidona (44). Quanto ao primeiro argumento, os estudos citados (37,38,65-70,78) , de eficácia e segurança, mostrando claras vantagens sobre todos os demais agentes, parecem não justificá-lo. Quanto ao segundo argumento, pode ser ele considerado, também, sem maior fundamento, como também foi afirmado por Keenan ${ }^{(71)}$, desde que a manipulação do produto seja efetuada por pessoal habilitado e responsável. Finalmente, quanto ao terceiro, se pensando em prevenção da CN, deve-se procurar utilizar o produto logo após o nascimento, com o objetivo de combater os agentes ainda na secreção ocular, adquirida na passagem pelo canal do parto. Qualquer agente deverá ser muito menos eficaz se for utilizado tardiamente.

O modo de usar consiste na aplicação de uma gota em cada olho logo após realização dos cuidados gerais e avaliação dos sinais vitais efetuados após o parto (inclusive a limpeza das pálpebras e dos olhos). A utilização de mais uma gota, cerca de 24 horas após o parto não mostrou vantagens em relação à aplicação de apenas uma gota ${ }^{(78)}$.

Estudo de Najafi ${ }^{(66)}$ comprova a estabilidade da iodopovidona a 2,5\% por vários meses, mas, após a abertura do frasco, o Formulário Nacional da ANVISA ${ }^{(73)}$ recomenda que a solução deve ser desprezada em 30 dias, o mesmo devendo ser feito se houver alteração na sua coloração ou no odor. 
Com relação ao uso da iodopovidona no Brasil, embora a ANVISA tenha se referido a ele em 2000 e $2005^{(72,73)}$, não se encontrou nenhuma recomendação oficial para que seja feito. Em outra publicação do MS, de $2006^{(1)}$, a possibilidade do uso nem sequer é citada. Com relação a outras referências nacionais, em trabalho publicado em $2007^{(74)}$, ele é fortemente recomendado. Em outro trabalho mais recente, de $2009^{(44)}$, informa que em 2007 a iodopovidona era utilizada em duas das cinco maternidades pesquisadas em Florianópolis, mas as vantagens do produto em relação aos demais não são ressaltadas.

Enfim, como se tem a informação extraoficial de que a iodopovidona não é utilizada na maioria das maternidades do Brasil, o objetivo desse trabalho é de alertar para a falta de padronização e adequação dos métodos de prevenção da $\mathrm{CN}$. Visa atingir os colegas que possam ter influência na Saúde Pública de seus Estados, de modo que avaliem os métodos de prevenção que estão sendo usados e que possam sugerir a utilização do método que, dentro dos conhecimentos atuais, deve ser o mais adequado, a iodopovidona a $2,5 \%$. Após essa comprovação, através de profundo estudo, realizado pelo seu setor de Farmácia, em atendimento às argumentações dos autores, a Secretaria de Saúde do Estado do Espírito Santo determinou a utilização da iodopovidona a $2,5 \%$ como método oficial de prevenção da $\mathrm{CN}$ em toda a rede pública e conveniada, o que, sem dúvida será um fator determinante na propagação do método para todas as maternidades, inclusive as privadas e do Estado. Essas últimas estão sendo comunicadas oficialmente sobre a conveniência da adoção do método e já estão tomando providências nesse sentido.

\section{Abstract}

Nowadays the use of drugs for prevention of neonatal conjunctivitis (NC) has been questioned in some developed countries, due to the high level of prenatal care. In Brazil, although no official data on the occurrence of NC is available, several recent studies has shown high prevalence of genital infection in childbearing age and pregnant women. This, coupled with the $30 \%$ to $50 \%$ transmission rate of genital chlamydia and gonococcus from mother to newborn, leads to the conclusion that chemoprophylaxis is justified. The potential for local and systemic complications and the need for laboratory tests for its diagnosis have made $N C$ an important public health problem, overlooked in Brazil, where there is no standardization of the method of prevention. Although the use of silver nitrate still appears to be the official method, it has been questioned due to incomplete protection against chlamydia, nowadays the leading agent of $\mathrm{NC}$, and by the frequent occurrence of chemical conjunctivitis. So, it has been replaced by other agents such as erythromycin, tetracycline and other antibiotics. The advantages of povidone-iodine compared to these agents in the various items analyzed, has suggested that it is the best, among the products tested so far, for the prevention of $N C$.

Keywords: Ophthalmia neonatorum; Conjunctivitis; Primary prevention; Silver nitrate; Sexually transmitted diseases; Chlamydia trachomatis; Neisseria gonorrhoe; Povidone-Iodine

\section{REFERÊNCIAS}

1. Brasil. Ministério da Saúde. Secretaria de Vigilância em Saúde. Programa Nacional de DST e AIDS. Manual de controle das doenças sexualmente transmissíveis. 4a ed. Brasília: Ministério da Saúde; 2006. 140 p. (Série Manuais, N. 68).

2. Laga M, Meheus A, Piot P. Epidemiology and control of gonococcal ophthalmia neonatorum. Bull World Health Organ. 1989;67(5):471-7. Erratum in: Bull World Health Organ. 1990;68(5):690.

3. Credé CS. Die Verhütung der Augenentzündung der Neugeborenem. Arch Gynaekol. 1881;18: 367-70.

4. Credé CS. Prevention of inflammatory eye disease in the newborn. Bull World Health Organ. 2001;79(3):264-6.

5. Christian JR. Comparison of ocular reactions with the use of silver nitrate and erythromycin ointment in ophthalmia neonatorum prophylaxis. J Pediatr. 1960;57(1):55-60.

6. Nishida H, Risemberg HM. Silver nitrate ophthalmic solution and chemical conjunctivities. Pediatrics. 1975;56(3):368-73.

7. O'Hara M. Ophthalmia neonatorum. Pediatr Clin North Am. 1993;40(4):715-25. Review.

8. External Disease and Cornea: section 8, Infectious Disease of the External Eye. San Francisco: American Academy of Ophthalmology; 2006. p.176.

9. Fox KR, Golomb HS. Staphylococcal ophthalmia neonatorum and the staphylococcal scalded skin syndrome. Am J Ophthalmol. 1979;88(6):1052-5.

10. Arffa RC. Infectious conjunctivitis. In: Arffa RC, Grayson M. Grayson's diseases of the cornea. 4a ed. San Louis: Mosby; 1997. p. $125-56$.

11. Horn JE, Hammer MI, Falkow S, Quinn TC. Detection of Chlamydia trachomatis in tissue culture and cervical scrapings by in situ DNA hybridization. J Infect Dis. 1986;153(6):1155-9.

12. Hammerschlag MR, Cummings C, Roblin PM, Williams TH, Delke I. Efficacy of neonatal ocular prophylaxis for the prevention of chlamydial and gonococcal conjunctivitis. N Engl J Med. 1989;320(12):769-72.

13. Pediatric Ophthalmology and Strabismus: Section 6, 2006. San Francisco, CA: American Academy of Ophthalmology; 2006. p. 221-4.

14. Geller M, Suchmacher M, Nunes FP. Clamídia: parte 2. J Bras Med. 2003;85(4):15-22. 
15. World Health Organization. Global prevalence and incidence of selected curable sexually transmitted infections. Overview and estimates. Geneva: World Health Organization; 2001. [cited 2010 Aug 12]. Available from: http://www.who.int/hiv/ pub/sti/who_hiv_aids_2001.02.pdf .

16. Miranda AE, Szwarcwald CL, Peres RL, Page-Shafer K. Prevalence and risk behaviors for chlamydial infection in a population-based study of female adolescents in Brazil. Sex Transm Dis. 2004;31(9):542-6.

17. Codes JS, Cohen DA, Melo NA, Teixeira GG, Leal AS, Silva TJ, Oliveira MPR. Detecção de doenças sexualmente transmissíveis em ambientes clínicos e não clínicos na cidade de Salvador, Bahia, Brasil. Cad Saúde Pública $=$ Rep Public Health. 2006;22(2):325-34.

18. Oliveira FA, Pfleger V, Lang K, Heukelbach J, Miralles I, Fraga F, et al. Sexually transmitted infections, bacterial vaginosis, and candidiasis in women of reproductive age in rural Northeast Brazil: a population-based study. Mem Inst Oswaldo Cruz. 2007;102(6):751-6.

19. Barcelos MRB, Vargas PRM, Baroni C, Miranda AE. Infecções genitais em mulheres atendidas em Unidade Básica de Saúde: prevalência e fatores de risco. Rev Bras Ginecol Obstet. 2008;30(7):349-54.

20. Fernandes AMS, Daher G, Nuzzi RXP, Petta CA. Infecção por Chlamydia trachomatis e Neisseria gonorrhoeae em mulheres atendidas em serviço de planejamento familiar. Rev Bras Ginecol Obstet. 2009;31(5):235-40.

21. Jalil EM, Pinto VM, Benzaken AS, Ribeiro D, Oliveira EC, Garcia EG, et al. Prevalência da infecção por clamídia e gonococo em gestantes de seis cidades brasileiras. Rev Bras Ginecol Obstet. 2008;30(12):614-9.

22. Leite RCS. Infecção cervical causada por Chlamydia trachomatis $e m$ gestantes. Estudo de prevalência e fatores de risco. Rev Bras Ginecol Obstet. 2001;23(1):58.

23. Araújo RSC. Estudo da infecção genital por Chlamydia trachomatis em adolescentes e jovens do sexo feminino no distrito sanitário leste do município de Goiânia: prevalência e fatores de risco. Rev Bras Ginecol Obstet. 2002;24(7):492.

24. Eleutério RMN, Eleutério Júnior J, Giraldo PC, Muniz AMV. Cervicite por Chlamydia trachomatis em mulheres sexualmente ativas atendidas em um serviço privado de ginecologia na cidade de Fortaleza. Rev Bras Anal Clin. 2007;39(4):287-90.

25. Centers for Disease Control. 1989 Sexually Transmitted Diseases Treatment Guidelines. MMWR Morb Mortal Wkly Rep. 1989;38 Suppl 8:1-43. Erratum in: MMWR Morb Mortal Wkly Rep. 1989;38(38):664.

26. Centers for Disease and Control and Prevention, Workowski KA, Berman SM. Sexually transmitted diseases treatment guidelines, 2006. MMWR Recomm Rep. 2006;55(RR-11):194. Erratum in: MMWR Recomm Rep. 2006;55(36):997.

27. Iyamu E, Enabulele O. A survey on ophthalmia neonatorum in Benin City, Nigeria (Emphasis on gonococcal ophthalmia). Online J Heath Allied Scs. 2003;2:2. [cited 2010 Aug.]. Avialable from: http://www.ojhas.org/issue6/2003-2-2.htm .

28. MacDonald N, Mailman T, Desai S. Gonococcal infections in newborns and in adolescents. Adv Exp Med Biol. 2008;609:108-30. Review.

29. Gilbert C, Foster A. Childhood blindness in the context of VISION 2020 - the right to sight. Bull World Health Organ. 2001;79(3):227-32.
30. Isenberg SJ, Apt L, Yoshimori R, McCarty JW, Alvarez SR. Source of the conjunctival flora at birth and implications for ophthalmia neonatorum prophylaxis. Am J Ophthalmol. 1988;106(4):458-62.

31. São Paulo (Estado). Decreto $N^{\circ} 9.713$, de 19 de abril de 1977. Aprova Norma Técnica Especial relativa à preservação da saúde, dispondo sobre a instilação obrigatória da solução de nitrato de prata a $1 \%$ nos olhos dos recém-nascidos (Método de Credé) [texto na Internet]. [citado 2010 Ago 12]. Disponível em: http://www.jusbrasil.com.br/legislacao/212246/ decreto-9713-77-sao-paulo-sp. c

32. São Paulo (Estado). Decreto No 19.941 , de 19 de novembro de 1982. Altera a redação dos itens 1,3 e 4 do inciso I da Norma Técnica Especial aprovada pelo Decreto $\mathrm{n}^{\circ}$ 9.713, de 19 de abril de 1977. [ Internet]. [citado 2010 Ago 12]. Disponível em:

http://www.jusbrasil.com.br/legislacao/199693/decreto-1994182-sao-paulo-sp

33. Pagliuca LMF, Fialho AVM, Simas ZA, Silva RPM. Agentes químicos profiláticos da oftalmia neonatal: estudo exploratório. Cogitare Enferm. 1997;2(1):18-20.

34. Silva EB, Alves KMB. Avaliação do uso do Método de Credé nas Casas de Parto e Maternidades da cidade de Fortaleza. Rev Bras Oftalmol. 1996;55(2):59-64.

35. Chambers HF. Antimicrobianos. In: Goodman LS, Gilman AG. Goodman \& Gilman: as bases farmacológicas da terapêutica. 10a ed. Rio de Janeiro: Guanabara Koogan; 2001. p. 929-53.

36. The United States Pharmacopeia: USP 21. 21a ed. Rockville: United States Pharmacopeial Convention; 1985.

37. Benevento WJ, Murray P, Reed CA, Pepose JS. The sensitivity of Neisseria gonorrhoeae, Chlamydia trachomatis, and herpes simplex type II to disinfection with povidone-iodine. Am J Ophthalmol. 1990;109(3):329-33.

38. Isenberg SJ, Apt L, Yoshimori R, Leake RD, Rich R. Povidone-iodine for ophthalmia neonatorum prophylaxis. Am J Ophthalmol. 1994;118(6):701-6.

39. Belfort R Jr, Imamura PM, Bonomo PP. Evaluación de la profiláxis de la oftalmia neonatal en la ciudad de São Paulo, Brasil. Bol Ofic Sanit Panam. 1975;78(3):212-20.

40. Palazzi MA, Munoz EH, Massaini MG, Aurichio MT, Barros OM. Estudo da aplicabilidade do método de Credé, em maternidades do município de São Paulo. Rev Bras Oftalmol. 1991;50(2):39-44.

41. Moreira L, Moreira H, Pires EF, Moreira CA. Avaliação do método de Credé nos serviços de maternidade de Curitiba. Rev Bras Oftalmol. 1993;52(2):47-50.

42. Netto LT, Netto DT, Fayad FA, Nassaralla Júnior JJ. Avaliação da aplicação do método de Credé em Goiânia - Goiás. Rev Bras Oftalmol. 1997;56(5):333-7.

43. Netto AA, Simas AZ. Avaliação do uso do Método de Credé em maternidades da Grande Florianópolis. Rev Bras Oftalmol. 1999;58(6):477-82.

44. Netto AA, Goedert ME. Avaliação da aplicabilidade e do custo da profilaxia da oftalmia neonatal em maternidades da grande Florianópolis. Rev Bras Oftalmol. 2009;68(5):264-70.

45. Thompson R, Isaacs ML, Khorazo D. A laboratory study of some antiseptics with reference to ocular application. Am J Ophthalmol. 1937;20:1087-99. 
46. Napchan BM, Morales RP, Carvalho ML, Cunha KV, Figueras A. From suspicion to action: the chemical conjunctivitis and silver nitrate connexion example in Brazilian hospitals. Pharmacoepidemiol Drug Saf. 2005;14(8):555-9.

47. Mathieu PL Jr. Comparison study: silver nitrate and oxytetracycline in newborn eyes; a comparison of the incidence of conjunctivitis following the instillation of silver nitrate or oxytetracycline into the eyes of newborn infants. AMA J Dis Child. 1958;95(6):609-11.

48. Giffin RB Jr. Eye damage in newborns from use of strong silver nitrate solutions. Calif Med. 1967;107(2):178-81.

49. Laga M, Plummer FA, Piot P, Datta P, Namaara W, Ndinya-Achola JO, et al. Prophylaxis of gonococcal and chlamydial ophthalmia neonatorum. A comparison of silver nitrate and tetracycline. $\mathrm{N}$ Engl J Med. 1988;318(11):653-7.

50. Zanoni D, Isenberg SJ, Apt L. A comparison of silver nitrate with erithromycin for prophylaxis against ophthalmia neonatorum. Clin Pediatr (Phila). 1992;31(5):295-8.

51. Chen JY. Prophylaxis of ophthalmia neonatorum: comparison of silver nitrate, tetracycline, erythromycin and no prophylaxis. Pediatr Infect Dis J. 1992;11(12):1026-30.

52. Bell TA, Grayston JT, Krohn MA, Kronmal RA. Randomized trial of silver nitrate, erythromycin, and no eye prophylaxis for the prevention of conjunctivitis among newborns not at risk for gonococcal ophthalmitis. Eye Prophylaxis Study Group. Pediatrics. 1993;92(6):755-60.

53. Hedberg K, Ristinen TL, Soler JT, White KE, Hedberg CW, Osterholm MT, MacDonald KL. Outbreak in erythromycinresistant staphylococcal conjunctivitis in a newborn nursery. Pediatr Infect Dis J. 1990;9(4):268-73.

54. Schwarcz SK, Zenilman JM, Schnell D, Knapp JS, Hook EW 3rd, Thompson S, et al. National surveillance of antimicrobial resistance in Neisseria gonorrhoeae. The Gonococcal Isolate Surveillance Project. JAMA. 1990;264(11):1413-7. Comment in: JAMA. 1990;264(11):1451-2.

55. Sánchez Luna M, Pallás Alonso C, Botet Mussons F, Echániz Urcelay I, Castro Conde J, Narbona E. Recomendaciones para el cuidado y atención del recién nacido sano en el parto y en las primeras horas después del nacimiento. An Pediatr (Barc). 2009;71(4):349-61.

56. Dipiro JT, Talbert R, Yee G, Matzke G, Wells B, Posey LM, editors. Pharmacotherapy: a pathophysiologic approach. 5th ed. New York: McGraw-Hill; c2002.

57. World Health Organization. Guidelines for the management of sexually transmitted infections. 2003. [cited 2010 Aug.]. Available from: http://whqlibdoc.who.int/publications/2003/ 9241546263.pdf

58. Sexually transmitted diseases treatment guidelines 2002. Centers for Disease Control and Prevention. MMWR Recomm Rep. 2002;51(RR-6):1-78.

59. Guidance about shortage of erythromycin $(0,5 \%)$ ophthalmic ointment. 31 ago 2009 [Letter]. Department of Health \& Human Services. Centers for Disease Control and Prevention. [cited 2010 Aug 12]. Avialable from: http://www.cdc.gov/ std/treatment/DCL-ErythromycinOintementShortage-8-312009.pdf

60. Centers for Disease Control and Prevention. Sexually Transmitted Diseases. CDC Guidance on Shortage of
Erythromicyn $(0.5 \%)$ Ophthalmic Ointment CDC Guidance on Shortage of Erythromicyn (0,5\%) Ophthalmic Ointment: September 2009. CDC - Centers for Disease Control and Prevention. [cited $2010 \mathrm{Au}$ 12]. Available from:www.cdc.gov/ std/treatment/2006/erythromycinOintmentShortage.htm

61. Canadian Paediatric Society (CPS). Recommendations for prevention of neonatal ophthalmia. Infectious Diseases and Immunization Committee. Last updated: March 2008. Reaffirmed in January 2009. [cited 2010 Aug 12]. Available from: http://www.cps.ca/english/statements/ID/ID02-03.htm.

62. Apt L, Isenberg S, Yoshimori R, Paez JH. Chemical preparation of the eye in ophthalmic surgery. III. Effect of povidone-iodine on the conjunctiva. Arch Ophthalmol. 1984;102(5):728-9.

63. Isenberg SJ, Apt L, Yoshimori R, Khwarg S. Chemical preparation of the eye in ophthalmic surgery. IV. Comparison of povidone-iodone on the conjunctiva with a prophylactic antibiotic. Arch Ophthalmol. 1985;103(9):1340-2.

64. Speaker MG, Menikoff JA. Prophylaxis of endophthalmitis with topical povidone-iodine. Ophthalmology. 1991;98(12):1769-75.

65. Isenberg SJ, Apt L, Wood M. A controlled trial of povidoneiodone as prophylaxis against ophthalmia neonatorum. $\mathrm{N}$ Engl J Med. 1995;332(9):562-6. Comment in: N Engl J Med. 1995;333(2):127; author reply 129. N Engl J Med. 1995;333(2):126; author reply 127. N Engl J Med. 1995;333(2):126-7. N Engl J Med. 1995;332(9):600-1.

66. Najafi RB, Samani SM, Pishya N, Moheimani F. Formulation and clinical evaluation of povidone-iodine ophthalmic drop. Iran J Pharm Res. 2003;2:157-160.

67. Ali Z, Khadije D, Elahe A, Mohammad M, Fateme Z, Narges Z. Prophylaxis of ophthalmia neonatorum comparison of betadine, erythromycin and no prophylaxis. J Trop Pediatr. 2007;53(6):388-92.

68. Houang ET, Gilmore OJ, Reid C, Shaw EJ. Absence of bacterial resistance to povidone iodine. J Clin Pathol. 1976;29(8):752-5.

69. Prince HN, Nonemaker WS, Norgard RC, Prince DL. Drug resistance studies with topical antiseptics. J Pharm Sci. 1978;67(11):1629-31.

70. Richter R, Below H, Kadow I, Kramer A, Müller C, Fusch C. Effect of topical $1.25 \%$ povidone-iodine eyedrops used for prophylaxis of ophthalmia neonatorum on renal iodine excretion and thyroid-stimulating hormone level. J Pediatr. 2006;148(3):401-3

71. Keenan JD, Eckert S, Rutar T. Cost analysis of povidoneiodine for ophthalmia neonatorum prophylaxis. Arch Ophthalmol. 2010;128(1):136-7.

72. Brasil. Ministério da Saúde. Agência Nacional de Vigilância Sanitária. Curso básico de controle de infecção hospitalar. Caderno C: métodos de proteção anti-infecciosa. 2000. [citado 2010 Set 23]. Disponível em: http://www.cvs.saude.sp.gov.br/ pdf/CIHCadernoC.pdf .

73. Brasil. Ministério da Saúde. Agência Nacional de Vigilância Sanitária. Resolução RDC n. 222, de 29 de julho de 2005. Aprova a $1^{\text {a }}$ Edição do Formulário Nacional, elaborado pela Subcomissão do Formulário Nacional, da Comissão Permanente de Revisão da Farmacopéia Brasileira (CPRVD), instituída pela Portaria $n^{\circ} .734$, de 10 de outubro de 2000. [citado 2010 Set 23]. Disponível em : http://anvisa.gov.br/e-legis/. 
74. Passos AF, Zabzinski L. Queimadura ocular pelo nitrato de prata. Rev Bras Oftalmol. 2007;66 (3):191-6.

75. Espírito Santo. Secretaria de Estado da Saúde. Portaria $n^{\circ}$ 005-R, de 26/01/2010, Diário Oficial dos Poderes do Estado. 27 de Janeiro de 2010, p. 15. [citado 2010 Ago. 20]. Disponível em: http://200.165.59.196/ farmaciacidada/_ midias/ pdf/3164b67196c94f15.pdf

76. Endriss D, Ventura LMVO, Diniz JR, Celino AC, Toscano J. Doenças oculares em neonatos. Arq Bras Oftalmol. 2002;65(5):551-5.

77. Ventura LMO, Travassos S, Ventura AG, Trigueiro L, Marques S. Um programa baseado na triagem visual de recém-nascidos em maternidades: Fundação Altino Ventura/2000. Arq Bras Oftalmol. 2002;65(6):629-35.

78. Isenberg SJ, Apt L, Del Signore M, Gichuhi S, Berman NG. A Double application approach to ophthalmia neonatorum prophylaxis. Br J Ophthalmol. 2003;87(12):1449-52. Comment in: Br J Ophthalmol. 2003;87(12):1437. Br J Ophthalmol. 2004;88(10):1352; author reply 1352 .
79. Assadian O, Assadian A, Aspöck C, Hahn D, Koller W. Prophylaxis of ophthalmia neonatorum-a nationwide survey of the current practice in Austria. Wien Klin Wochenschr. 2002;114(5-6):194-9. Comment in: Wien Klin Wochenschr. 2002;114(5-6):171-2.

80. Schaller UC, Klauss V. Is Credé's prophylaxis for ophthalmia neonatorum still valid? Bull World Health Organ. 2001;79(3):262-3.

\section{Endereço para correspondência \\ Angelo Ferreira Passos}

Rua Alvim Soares Bermudes, $n^{\circ} 261$ - casa 13

Morada de Camburi

CEP 29062-515 - Vitória (ES), Brasil

e-mail: angelopassos@terra.com.br 